\title{
Heisenberg spin chains with additional isotropic three-site exchange interactions
}

\begin{abstract}
N.B. Ivanov
Institute of Solid State Physics, Bulgarian Academy of Sciences, Tzarigradsko chaussée 72, 1784 Sofia, Bulgaria

Received June 15, 2020, in final form July 10, 2020

The $J_{1}-J_{3}$ Heisenberg spin models with nearest-neighbor $\left(J_{1}\right)$ and additional isotropic three-site $\left(J_{3}\right)$ spin interactions remain relatively less explored, although such types of competing exchange terms can naturally emerge from different sources, including the strong-coupling expansion of the multiorbital Hubbard model. Below we present a short survey of the recently published research in this field, the emphasis being on the characteristics of the variety of quantum phases supported by a few generic uniform- and alternating-spin $J_{1}-$ $J_{3}$ Heisenberg chains. For the reason that the positive $\left(J_{3}>0\right)$ three-site couplings tend towards the formation of local quantum dimers, the $J_{1}-J_{3}$ spin models typically experience some spontaneous dimerization upon increasing $J_{3}$. Actually, it occurred that the established dimer phases in spin- $S J_{1}-J_{3}$ Heisenberg chains $(S>1 / 2)$ serve as complete analogues of the famous gapped Majumdar-Ghosh dimer phase in the spin-1/2 Heisenberg chain with next-nearest-neighbor couplings. The same dimerizations have been observed in the alternating-spin $(S, \sigma) J_{1}-J_{3}$ chains $(S>\sigma)$, provided that the cell spin $S+\sigma=$ integer, whereas for halfinteger cell spin, the local dimer formation produces gapless spin-liquid ground states. The alternating-spin $J_{1}-J_{3}$ chains also provide some typical examples of spin models supporting the so-called non-Lieb-Mattis magnetic phases.
\end{abstract}

Key words: spin chains, quantum spin phases, three-spin interactions

\section{Introduction}

Over the past two decades, it has been established that the Heisenberg spin systems with additional competing interactions - such as longer-range exchange bonds, Dzyaloshinskii-Moria couplings, as well as ring and biquadratic exchange couplings - support a rich variety of spin phases, including the exotic spin ice and spin nematic states, as well as various spin liquids [1]. In particular, the phase diagrams of Heisenberg spin models with the two-site biquadratic exchange (2BE) term $\left(\boldsymbol{S}_{i} \cdot \boldsymbol{S}_{j}\right)^{2}$, where $\boldsymbol{S}_{i}$ and $\boldsymbol{S}_{j}$ are lattice spins $\left(\left|\boldsymbol{S}_{i}\right|,\left|\boldsymbol{S}_{j}\right|>1 / 2\right)$, were extensively studied, typical examples being the spin-1 bilinear-biquadratic chain [2] and its higher-dimensional counterparts on square [3, 4], triangular [5, 6], and cubic [3] lattices.

At the same time, by now the effect of the isotropic three-site exchange (3SE) terms

$$
\left(\boldsymbol{S}_{i} \cdot \boldsymbol{S}_{j}\right)\left(\boldsymbol{S}_{i} \cdot \boldsymbol{S}_{k}\right)+\text { H.c. } \quad\left(\left|\boldsymbol{S}_{i}\right|>1 / 2, \quad i \neq j, \quad k, j \neq k\right)
$$

on Heisenberg spin systems remains relatively less explored. As a matter of fact, often both higher-order exchange terms appear on an equal footing, for example, in the fourth-order strong-coupling expansion of the two-orbital Hubbard model [7], so that finding higher-order exchange effects in real materials could be a challenge. Indeed, to the best of our knowledge, the only more or less convincing experimental evidence for 3SE effects comes from inelastic neutron scattering results for the low-lying excitations in the magnetic material $\mathrm{CsMn}_{x} \mathrm{Mg}_{1-x} \mathrm{Br}_{3}(x=0.28)$ [8], $\mathrm{CsMnBr}_{3}$ being known as a nearly ideal isotropic one-dimensional (1D) Heisenberg antiferromagnet with site spins $S=5 / 2$. These experimental results predict almost identical strengths of both higher-oder exchange terms (2BE and 3SE), which are about two orders of magnitude weaker than the principal bilinear Heisenberg coupling. It was established that 
the higher-order exchange interactions in $\mathrm{CsMn}_{x} \mathrm{Mg}_{1-x} \mathrm{Br}_{3}$ also appear as a result of magnetoelastic forces [9]. Similar magnetostriction effects — discussed earlier for polynuclear complexes of iron-group ions [10] - were predicted for some single-molecular magnets [11].

In both mentioned scenarios for creating higher-oder exchange terms, 2BE and 3SE interactions are controlled by one and the same parameter, so that it might be difficult to separate their effects in the experiment. Cold atoms in optical lattices open a promising route in this direction. It was demonstrated [12-14] that with the two-species Bose-Hubbard model in a triangular configuration, a wide range of Hamiltonian operators can be generated, including different three-spin interactions. The latter are due to the possibility of atomic tunneling through different paths from one vertex to the other, and can be extended to 1D spin models with three-spin interactions. Another intriguing system in optical lattices concerns polar molecules driven by microwave fields, naturally giving rise to Hubbard models with strong nearest-neighbor three-site interactions [15]. Since the two-site bilinear terms can be separately turned with external fields, this system opens a promising route for experimental studies of such higher-order exchange interactions. Let us also note that if one of the spins in the $2 \mathrm{BE}$ interaction is a spin- $1 / 2$ operator, then this term reduces to an isotropic bilinear exchange. Thus, in some alternating-spin Heisenberg systems, the 2BE terms can be in principle excluded.

On the theoretical side, generalized Heisenberg chains with additional 3SE terms have already been discussed in the literature, mostly as a tool to construct various isotropic [16-21] as well as spin$1 / 2$ anisotropic [22-30] exactly-solvable spin models. Note that the spin systems with anisotropic spin interactions go beyond the scope of the present survey, so that we restrict ourselves to a few comments. As mentioned above, in the case of spin- $1 / 2$ operators, the expression in equation (1.1) reduces to a bilinear two-site term. Therefore, the 3SE interaction should necessarily contain products of three spin-1/2 operators defined on separate lattice sites. Clearly, such additional terms violate the time and, eventually, the space reversal symmetries, so that they might be expected to produce quite different effects as compared to the isotropic case. Actually, it was demonstrated that the discussed peculiarities of the $3 \mathrm{SE}$ terms in spin- $1 / 2$ chains can produce a number of intiguing effects, including the support of some specific phases such as the chiral [23], weak-ferromagnetic [26], as well as ferroelectric [30] spin states.

Turning again to the case of Heisenberg spin chains with isotropic 3SE interactions, note that only in the recent few years the quantum phase diagrams of such models with arbitrary strengths $\left(J_{3}\right)$ of the 3SE couplings were discussed in the literature. Here, we present a short survey of the recent research in this field, the emphasis being on a few generic 1D Heisenberg spin models with extra 3SE couplings, including the spin-1 and spin-3/2 uniform chains [31-37], as well as the mixed-spin $(1,1 / 2)$ and $(3 / 2,1 / 2)$ chains [38-41]. The interest in such generalized $\left(J_{1}-J_{3}\right)$ isotropic spin models is partially motivated by the belief that the competing 3SE interactions could produce specific phase diagrams, which are not typical of spin systems defined on frustrated lattices and/or with extra well-studied competing interactions such as the longer-ranged exchange bonds and the 2BE interactions. Actually, as discussed below, the $3 \mathrm{SE}$ couplings exhibit some unique features - like the promotion of collinear classical spin configurations, or the reinforcing tendency towards clustering of the quantum spins on the shortest exchange bonds which may stabilize some new spin phases.

The survey is organized as follows. In section 2 we discuss recent works related to the quantum phase diagrams of two spin- $S$ Heisenberg chains $(S=1$ and 3/2) and extra isotropic 3SE interactions. The same issues, but addressed to the alternating-spin Heisenberg chains with extra isotropic 3SE couplings and site spins $(S, \sigma)=(1,1 / 2)$ and $(3 / 2,1 / 2)$, are discussed in section 3 . Most of the presented numerical results were obtained using the density-matrix renormalization group (DMRG) and exact numerical diagonalization (ED) techniques. The last section contains conclusions and some prospects for future developments in the field.

\section{Spin-S $J_{1}-J_{3}$ Heisenberg chains [31-37]}

The Heisenberg spin- $S$ chain with additional isotropic 3SE interactions is defined by the Hamiltonian

$$
\mathcal{H}_{1-3}=\sum_{i=1}^{N}\left\{J_{1} \boldsymbol{S}_{i} \cdot \boldsymbol{S}_{i+1}+J_{3}\left[\left(\boldsymbol{S}_{i-1} \cdot \boldsymbol{S}_{i}\right)\left(\boldsymbol{S}_{i} \cdot \boldsymbol{S}_{i+1}\right)+\text { H.c. }\right]\right\},
$$


where $S_{i}(i=1,2, \ldots, N)$ are site spins characterized by the quantum spin number $S>1 / 2, N$ being the number of lattice sites. In the extreme quantum case $S=1 / 2$, the $J_{3}$ term reduces to a bilinear nextnearest-neighbor (NNN) isotropic exchange interaction, as the squaring of any Pauli matrix gives the unit matrix. As a matter of fact, the most extreme quantum case with non-trivial $J_{3}$ terms can be realized in spin systems with exchange bonds connecting only spin- 1 and spin- $1 / 2$ operators, the alternating-spin models discussed in the next section being perhaps the simplest and realistic examples of this kind. For convenience, we use the following parameterization $J_{1}=J \cos \theta$ and $J_{3}=J \sin \theta$, where $\theta \in[0,2 \pi]$, and without loss of generality we set $J=1$.

\subsection{Spin-1 $J_{1}-J_{3}$ Heisenberg chain}

One of the first intriguing results, concerning $J_{1}-J_{3}$ Heisenberg chains with an arbitrary site spin $S$, states that for some strengths of the $J_{3}$ interaction, i.e., $J_{1} / J_{3}=[4 S(S+1)-2]$, the spin-S chain, equation (2.1), exhibits an exact fully-dimerized (i.e., Majumdar-Ghosh type) ground state (GS) [31]. This statement was soon generalized for $J_{1}-J_{3}$ Heisenberg chains with additional NNN and alternating-bond interactions [33]. Using the conformal field-theory and DMRG numerical simulations, Chepiga et al. further analyzed the spontaneous dimerization transition in the spin- $1 J_{1}-J_{3}$ chain with additional NNN bonds [34-36].

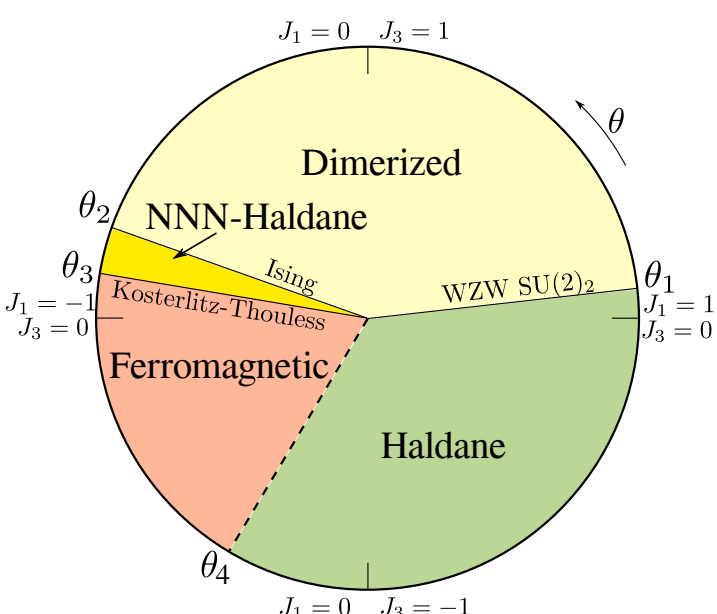

(a)

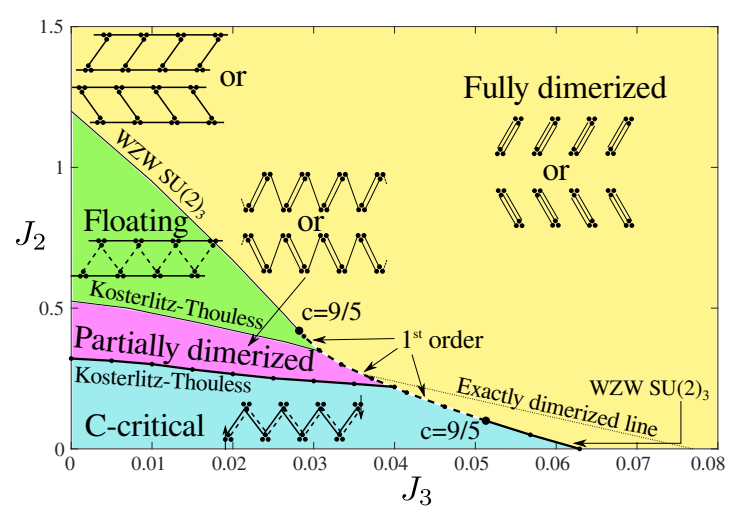

(b)

Figure 1. (Colour online) (a) Phase diagram of the spin-1 Heisenberg chain with extra isotropic 3SE interaction $\left(J_{1}-J_{3}\right.$ model) defined by equation 2.1 . $\theta_{1} \approx 0.03519 \pi, \theta_{2} \approx 0.8913 \pi, \theta_{3} \approx 0.9474 \pi$, $\theta_{4} \approx 1.33 \pi[35]$. (b) Phase diagram of the spin-3/2 Heisenberg chain with next-nearest-neighbor $\left(J_{2}\right)$ and 3SE $\left(J_{3}\right)$ interactions. The dimerized phases are gapped, whereas the C-critical and the floating phases are characterized by a gapless spectrum. In terms of valence-bond states (VBS), the fully dimerized phase is represented as three valence bonds (solid lines) on every other $J_{1}$ bond, the partially polarized phase is described by alternating one and two valence bonds, whereas the critical phase is visualized as one valence bond that resonates between two neighboring bonds (dashed lines) [37].

Turning to the quantum phase diagram of the spin- $1 J_{1}-J_{3}$ Heisenberg chain, figure 1 (a), we see that the spin system supports four different phases in the interval $\theta \in[0,2 \pi]$, i.e., Haldane, dimerized, NNNHaldane, and ferromagnetic (FM) phases. It was established that the transition between the Haldane and dimerized phases at $\theta_{1}$ is continuous and belongs to the Wess-Zumino-Witten (WZW) $S U(2)_{k}(k=2)$ universality class [31]. Close to the pase boundary $\theta_{1}$ there is a dimerized point precisely at $J_{3} / J_{1}=1 / 6$.

At $\theta_{2}$, an Ising-type transition takes place between the dimerized and the so-called NNN-Haldane phase [34, 35]. The latter is a spin-1 counterpart of the dimerized Majumdar-Ghosh phase in the spin-1/2 $J_{1}-J_{2}$ Heisenberg chain $-J_{2}$ being the strength of the NNN exchange bond - but it consists of two effectively decoupled NNN Haldane chains [42]. The DMRG data also suggest that in the thermodynamic limit the singlet-triplet bulk gap remains open at $\theta_{2}$, while the spectrum becomes critical within the singlet 
sector. The DMRG results imply a direct Kosterlitz-Thouless phase transition at $\theta_{3}$ between the NNNHaldane and the FM phases. On the other hand, the transition between the FM phase and the Haldane phase at $\theta_{4}$ is first order [36].

\subsection{Spin-3/2 $J_{1}-J_{3}$ Heisenberg chains}

In the spin-3/2 $J_{1}-J_{3}$ chain, the transition from the critical phase to a spontaneously dimerized state at $J_{3} / J_{1} \approx 0.063$ [32] is continuous and belongs to the $S U(2)_{k}(k=3)$ WZW universality class [43]. In figure 1 (b) we show the extended phase diagram of the spin-3/2 $J_{1}-J_{3}$ Heisenberg chain with additional NNN terms of the form $S_{i-1} \cdot S_{i+1}$, which are controlled by the parameter $J_{2}$ [37].

The phase diagram consists of two dimerized phases (partially and fully dimerized) and two critical phases with commensurate and incommensurate correlations. Using the valence-bond singlets (VBS) picture, the fully dimerized phase corresponds to three valence bonds on every other $J_{1}$ bond, while the partially dimerized phase corresponds to alternating one and two valence bonds (see the insets in figure 1 ).

In terms of VBS singlets, the commensurate C-critical phase can be represented as one valence bond per $J_{1}$ bond, and, additionally, one resonating bond between two neighboring bonds (the dashed lines). By contrast, the other critical phase - called floating phase - appears for larger $J_{2}$. It is characterized by an incommensurate wave vector $q$ which changes within the phase area. Similar phases with a varying $q$ are supported by the mixed-spin $J_{1}-J_{3}$ chains, as well (see the next section). Further interesting details concerning the phase diagram of the extended spin- $3 / 2 J_{1}-J_{2}-J_{3}$ Heisenberg model can be found in the original work [37].

\section{Mixed-spin $J_{1}-J_{3}$ Heisenberg chains [38-41]}

In view of the numerous experimentally accessible quasi-1D systems described by the mixed-spin Heisenberg model (see, e.g., references [11] and [44]), it is instructive to analyze the following alternatingspin variant of the spin- $S J_{1}-J_{3}$ Heisenberg chain discussed above [38]

$$
\mathcal{H}_{1-3}^{\prime}=\sum_{n=1}^{L}\left\{J_{1} S_{2 n} \cdot\left(\sigma_{2 n-1}+\sigma_{2 n+1}\right)+J_{3}\left[\left(S_{2 n} \cdot \sigma_{2 n-1}\right)\left(S_{2 n} \cdot \sigma_{2 n+1}\right)+\text { H.c. }\right]\right\} .
$$

Here, $L$ stands for the number of elementary cells, each containing two different spins $(S>\sigma)$. We shall use again the standard parameterization of the coupling constants $J_{1}=J \cos t$ and $J_{3}=J \sin t$ $(0 \leqslant t<2 \pi)$, where $J=1$.

Since the effective strength of the extra term is controlled by the parameter $S \sigma J_{3}$, one expects that this interaction may play an important role especially in $(S, 1 / 2)$ chains and rings with large $S$ spins $(S \gg 1 / 2)$. In the extreme quantum case $(S, \sigma)=(1,1 / 2)$, it was demonstrated that the Hamiltonian $\mathcal{H}_{1-3}^{\prime}$ reproduces - up to irrelevant constants - the Hamiltonian of isotropic spin- $1 / 2$ diamond chain with an additional ring exchange in the plaquettes in the Hilbert subspace where the pairs of "up" and "down" plaquette spins form pure triplet states [45]. As mentioned above, the alternating-spin systems provide another realistic onset for separating the effects of the higher-order exchange interactions $2 \mathrm{BE}$ and 3SE. In particular, the Heisenberg chain with alternating $S$ and $\sigma=1 / 2$ spins $(S>1 / 2)$ provides a simple example of this kind. Indeed, according to the operator identity $\left(S_{i} \cdot \sigma_{j}\right)^{2} \equiv-S_{i} \cdot \sigma_{j} / 2+S(S+1) / 4$, the biquadratic terms in this system reduce to bilinear isotropic exchange terms.

\subsection{Classical phase diagram}

The classical phase diagram of the spin model (3.1) (inner circles in figure 3) can be constructed by using the GS cluster configurations shown in figure 2 By fitting the directions of the sharing $\sigma$ spins of the FM and FiM three-spin clusters, one can obtain macroscopic non-degenerate FM and FiM configurations corresponding to local minima of the classical energy, the FM and FiM phases in figure 3 . Using the two cluster configurations denoted as $D$, the same procedure leads to a $2^{L}$-fold degenerate 


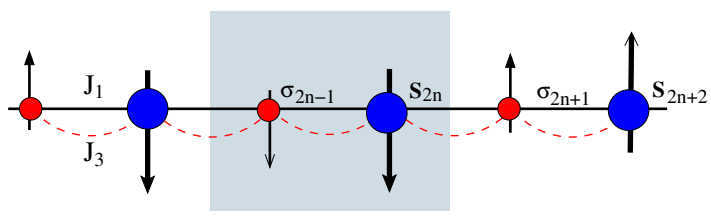

(a)

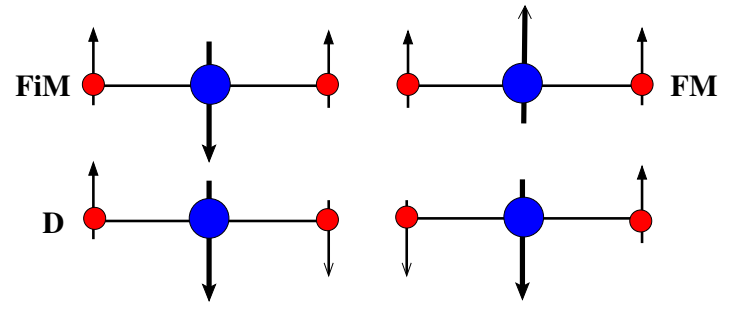

(b)

Figure 2. (Colour online) (a) Sketch of the mixed-spin Heisenberg chains and (b) the optimal cluster states used as building blocks of the classical spin phases presented in figure 3

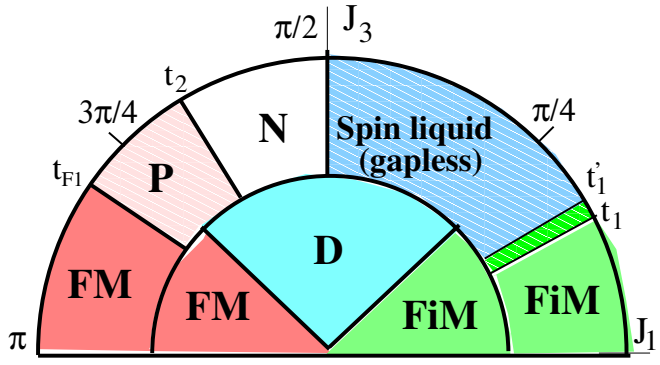

$\mathrm{S}=1, \sigma=1 / 2$

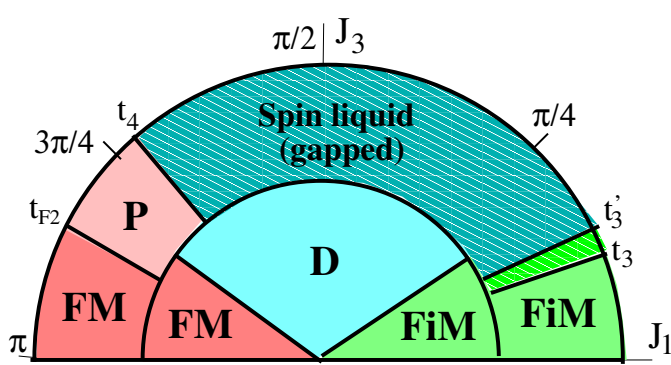

$\mathrm{S}=3 / 2, \sigma=1 / 2$

Figure 3. (Colour online) Classical (inner circles) and quantum (outer circles) phase diagrams of the $J_{1}-J_{3}$ mixed-spin chains with $(S, \sigma)=(1,1 / 2)$ (left-hand) and $(S, \sigma)=(3 / 2,1 / 2)$ (right-hand). FM and FiM denote the collinear ferromagnetic and ferrimagnetic phases, respectively. $\boldsymbol{P}$ is a partially polarized magnetic state with a spatially modulated magnetization. $\boldsymbol{D}$ denotes a $2^{L}$-fold degenerate classical phase composed of two types of collinear cluster spin configurations shown in figure 2 This phase contains both non-magnetic and magnetic ground-state configurations. The gapless/gapped spin liquid states correspond to chains with half-integer/integer cell spins $S+\sigma . N$ stands for a quasi-nematic state characterized by short-range nematic order and spin-2 lowest-lying excitations. Here, $t_{1} \approx 25.0^{\circ}$, $t_{1}^{\prime} \approx 30.0^{\circ}, t_{2} \approx 120.0^{\circ}, t_{3} \approx 20.1^{\circ}, t_{3}^{\prime} \approx 25.5^{\circ}$, and $t_{4} \approx 132.0^{\circ} . t_{\mathrm{F}}=\pi-\arctan [1 / \sigma(2 S+1)]$ is the exact FM phase boundary for arbitrary site spins $S$ and $\sigma\left(t_{\mathrm{F} 1} \approx 146.3^{\circ}, t_{\mathrm{F} 2} \approx 153.4^{\circ}\right)$. The classical phase boundaries between the FM and FiM phases of both models (not presented in this figure) are located at $t=3 \pi / 2$. In the $(1,1 / 2)$ case, quantum fluctuations stabilize a new doubly-degenerate (period two lattice cells) collinear magnetic phase in the interval $253.1^{\circ}<t<264.0^{\circ}$, whereas the boundary in the $(3 / 2,1 / 2)$ case remains unchanged [38, 40].

classical phase, denoted as $D$ phase in figure 3 . At a classical level, the phase diagrams of both models are qualitatively indistinguishable.

\subsection{Quantum phase diagrams}

\subsubsection{Modulated non-Lieb-Mattis type magnetic states}

In both models, the established partially-polarized magnetic states $(P$ and the narrow sectors close to the points $t_{1}$ and $t_{3}$ in figure 3 do not appear in the classical phase diagram. Due to the frustration effect of the three-site interaction, the Lieb-Mattis theorem [46] is not applicable, so that the so-called quantization of the unit-cell magnetic moment is destroyed. This means that the magnetic moment per cell can take arbitrary intermediate values. In particular, in such systems the magnetic moment may change continuously to zero near the transition from magnetic to non-magnetic states $\left(t_{1}^{\prime}\right.$ and $t_{3}^{\prime}$ points in figure 3), or to be spatially modulated, like in the $P$ sectors of both models close to the FM phase boundaries $t_{\mathrm{F} 1}$ and $t_{\mathrm{F} 2}$. In the latter case, the local magnetization clearly shows an incommensurate modulation with long-distance periodicity depending on the distance from the FM boundary. As a 


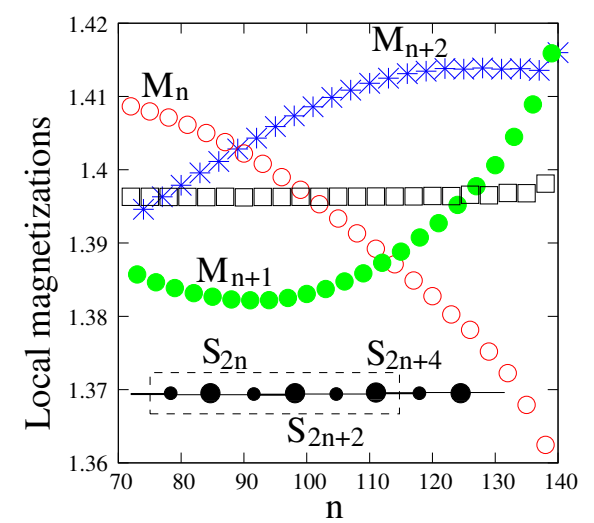

(a)

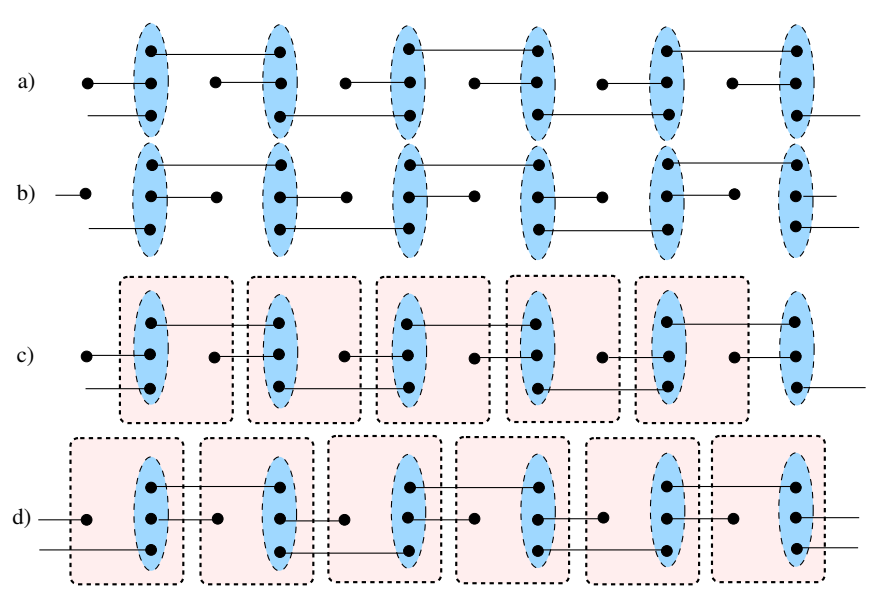

(b)

Figure 4. (Colour online) (a) The on-site magnetizations $M_{n}=\left\langle S_{2 n}^{z}\right\rangle$ in the $\mathrm{P}$ phase of the $(3 / 2,1 / 2)$ chain as functions of the cell index $n$ (DMRG, $t \approx 153.4^{\circ}, L=144$, open boundary conditions). Here, $n=3 j-2,3 j-1,3 j$ and $j=1,2, \ldots, L / 3$. Squares represent the constant average magnetization $\left(M_{n}+M_{n+1}+M_{n+2}\right) / 3$ including three neighboring unit cells. The results demonstrate the establishment of a periodic three-cell $(q=3)$ magnetic structure close to the FM transition point $t_{\mathrm{F} 2}$ in the P phase. The Inset shows the magnetic supercell containing six spins (i.e., three unit cells). (b) VBS picture of the doubly degenerate spin-liquid phase of the $(3 / 2,1 / 2)$ chain close to the phase boundaries $t_{3}^{\prime}(a, b)$ and $t_{4}(\mathrm{c}, \mathrm{d})$, respectively. Black dots denote spin- $1 / 2$ variables. The lines between two spins $1 / 2$ denote a singlet bond, whereas the dashed ellipses and rectangles denote symmetrization of the spin- $1 / 2$ variables. The first two (the last two) VBS states approximately represent ground states of the open spin-1 (spin-2) AFM Heisenberg chain. In the intermediate region, only a part of the composite cell spins form spin-2 states [40].

matter of fact, these modulated partially-polarized states follow the Oshikawa-Yamanaka-Affleck rule $q\left(S+\sigma-m_{0}\right)=$ integer [47], where $q$ is the period of the modulated structure, and $m_{0}$ is the magnetic moment per unit cell [38, 40]. Extremely close to the FM boundaries, $t \lesssim t_{\mathrm{F} 1}\left(t \lesssim t_{\mathrm{F} 2}\right)$, DMRG results imply that the modulated magnetic structures are characterized by $\left(q, m_{0}\right)=(8,9 / 8)$ and $(3,5 / 3)$ for the $(1,1 / 2)$ and $(3 / 2,1 / 2)$ models, respectively.

As an example, in figure 4 (a) we present the on-site magnetizations of the established $q=3$ magnetic structure in the alternating-spin $(3 / 2,1 / 2) J_{1}-J_{3}$ chain extremely close to the exact FM boundary $t_{\mathrm{F} 2}$, but out of the FM phase. As required for a plateau state, at this point the established magnetization per cell $m_{0}=5 / 3$ exactly fulfills the mentioned general rule for period $q=3$. Interestingly, the effect of the open boundaries in this case simply reduces to some local redistribution of the magnetic moment in the framework of the supercell, but the parameter $m_{0}$, characterizing the supercell as a whole, remains practically constant with the cell index $n$, excluding some narrow region near the system boundary. Skipping the further discussions on these exotic modulated partially-polarized states, we mention that such non-Lieb-Mattis-type magnetic phases in spin systems were originally identified in [45], and later were studied in a number of other 1D frustrated spin models [48, 49].

\subsubsection{Degenerated spin-liquid phases}

DMRG analysis of the short-range correlations in open chains reveals the regions in the parameter space where the lowest-energy states in both alternating-spin $\left(J_{1}-J_{3}\right)$ models exhibit a regular alternatingbond structure characterized by different values of the spin-spin correlators $\left\langle\sigma_{2 n-1} \cdot \boldsymbol{S}_{2 n}\right\rangle=u$ and $\left\langle\boldsymbol{S}_{2 n} \cdot \boldsymbol{\sigma}_{2 n+1}\right\rangle=v(u<v)$. The $u v(v u)$ dimerized GS $\left|\Psi_{\mathrm{L}}\right\rangle\left(\left|\Psi_{\mathrm{R}}\right\rangle\right)$ is stabilized in open chains with a $\sigma$ spin on the left (right) end of the chain and corresponds to a $u v(v u)$ dimerization. The established $u v$ structure of the GS is strongly revealed in the middle of the phase-diagram regions occupied by non-magnetic states of both models, where the values of $u$ and $v$ indicate the formation of nearly pure spin-1/2 and spin-2 (or spin-3) states of the nearest-neighbor spins in the systems with half-integer 
and integer cell spins $S+\sigma$, respectively, see figure 4(b). As mentioned above, similar dimerization effects of the $3 \mathrm{SE}$ interactions also appear in the spin-S $J_{1}-J_{3}$ Heisenberg chains, the NNN-Haldane phase being such a typical example. Since a given $\boldsymbol{S}_{2 n}$ spin can form such local dimer states in two different ways — including the left-hand or the right-hand nearest-neighbor $\sigma$ spin — all phases in the non-magnetic regions are doubly degenerate, irrespective of the low-energy structure of the spectrum (i.e., gapped or gapless). The finite-size scaling of the lowest triplet excitation in the alternating-spin $(1,1 / 2) J_{1}-J_{3}$ Heisenberg model suggests a gapless doubly-degenerate non-magnetic state — as may be expected from the dimerized structure of GS - constructed from local spin-1/2 dimers. On the other hand, the dimerized spin-liquid state of the alternating-spin $(3 / 2,1 / 2) J_{1}-J_{3}$ Heisenberg chain suggests the formation of either spin-1 (smaller $t$ ), or spin-2 (larger $t$ ) local dimers, which leads to the formation of a gapped doubly-degenerated spin-liquid state in the whole non-magnetic region of the phase diagram.

\subsubsection{Nematic-like phase}

The $J_{3}$ interaction term related to the $n$-th unit cell, equation (1.1), can be represented in the following symmetric form

$$
V_{n}^{(3)}=\frac{1}{2} \sum_{\alpha, \beta}\left(S_{2 n}^{\alpha} S_{2 n}^{\beta}+S_{2 n}^{\beta} S_{2 n}^{\alpha}\right)\left(\sigma_{2 n-1}^{\alpha} \sigma_{2 n+1}^{\beta}+\sigma_{2 n-1}^{\beta} \sigma_{2 n+1}^{\alpha}\right),
$$

where $\alpha, \beta=x, y$, and $z$. The two symmetric forms in the parentheses can be considered - up to some normalization factors - as tensor order parameters of on-site and bond spin nematic phases constructed from $S$ and $\sigma$ spins, respectively. Taking into consideration this form of $V_{n}^{(3)}$ let us speculate that for dominating isotropic $3 \mathrm{SE}$ interactions nematic orders of different types could be stabilized. As a matter

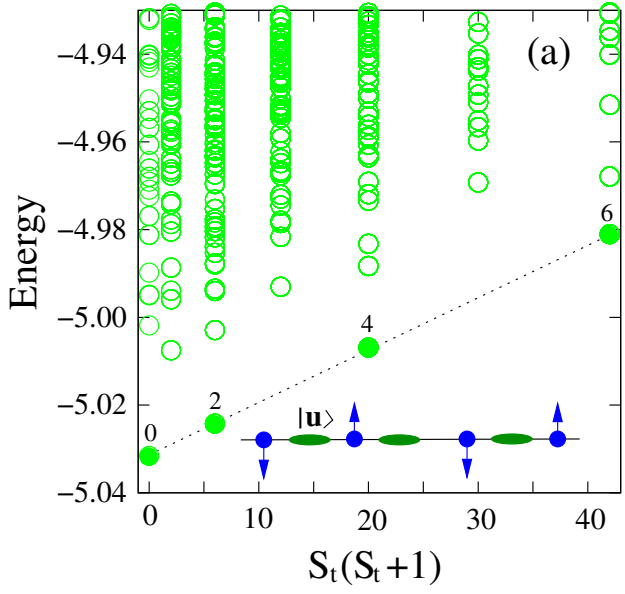

(a)

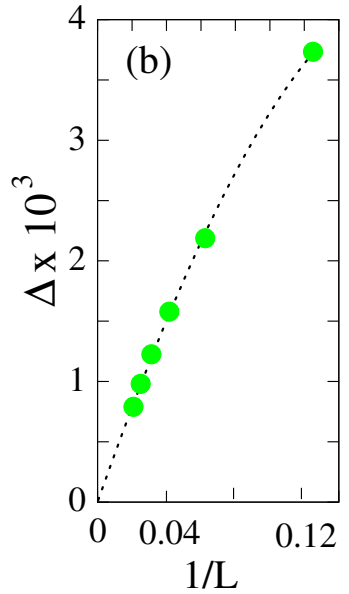

(b)

Figure 5. (Colour online) (a) Numerical ED results for the energy spectrum of the alternating-spin $(S=1$, $\sigma=1 / 2) \operatorname{ring}\left(L=8, t=110^{\circ}\right)$ vs $S_{\mathrm{t}}\left(S_{\mathrm{t}}+1\right)$, where $S_{\mathrm{t}}$ is the total spin. The lowest multiplets in the even $S_{\mathrm{t}}$ sectors (filled circles) form a tower of states with energies $E(S) \propto S_{\mathrm{t}}\left(S_{\mathrm{t}}+1\right)$. The dashed line is a guide to the eye. Inset: Cartoon of the suggested nematic-like state in the $\mathrm{N}$ sector constructed only with $S$ spins. The ellipses denote the local nematic states $|\boldsymbol{u}\rangle=\sum_{\alpha} u^{\alpha}|\alpha\rangle$, where $\boldsymbol{u}$ is a unit real vector and $|\alpha\rangle(\alpha=x, y, z)$ is the vector basis of the spin-1 operator $\boldsymbol{S}$. (b) Finite-size scaling of the gap to the lowest spin-2 excited state. This state is the lowest excited state in the $\mathrm{N}$ region. The dashed line denotes the least-squares fit to the DMRG data $\left(t=110^{\circ}, \mathrm{OBC}\right)$ obtained by the fitting ansatz $L \Delta(L)=a_{0}+a_{1} / L+a_{2} / L^{2}[38,39]$.

of fact, the numerical ED calculations concerning the lowest-lying excitations in periodic $L=8$ chains, described by the Hamiltonian (3.1), point towards the formation of some short-ranged nematic order only in the subsystem of $S$ spins [39], the $N$ sector in figure 3. Unfortunately, DMRG slowly diverges, so that it is not effective, in this region of the phase diagram. However, some valuable information on 
the non-magnetic $N$ state in the alternating-spin $(1,1 / 2) J_{1}-J_{3}$ Heisenberg model can also be extracted from the lowest-lying states in different total-spin $\left(S_{\mathrm{t}}\right)$ sectors. As well-known, the established tower of well-separated lowest multiplets, containing only even $S_{\mathrm{t}}$ sectors, is a fingerprint of the spectrum of quadrupolar states (i.e., spin nematic phase), unlike the Anderson tower of states [50] containing all $S_{\mathrm{t}}$ sectors. The latter is a characteristic of the Néel order. In fact, Anderson towers of states were observed even in some finite isotropic spin- $S$ chains and magnetic molecules [51]. Interestingly, such regions with enhanced quadrupolar fluctuations do not appear on the phase diagram of the $(3 / 2,1 / 2)$ model. Clearly, the discussed non-magnetic phase deserves further studies.

\section{Conclusion}

We discussed the quantum phase diagrams of 1D isotropic spin models with extra three-spin interactions, the emphasis being on a few generic spin-1 and alternating-spin $\left(J_{1}-J_{3}\right)$ Heisenberg chains. As a whole, the models are characterized by rich phase diagrams, most of the phases being connected with some special features of the 3SE interaction, equation (1.1). For example, a typical characteristic of the discussed models is the presence of spontaneously dimerized phases in the upper part of the phase diagram $\left(J_{3}>0\right)$. Clearly, this can be related to the fact that for positive $J_{3}$ the 3 SE terms promote the formation of local quantum dimers.

Another interesting feature of the studied diagrams is the appearance of some spatially modulated (non-Lieb-Mattis) partially-polarized states between the collinear (FM and FiM) magnetic phases and the spin-liquid states in the alternating-spin models. Note that neither spiral nor spatially-modulated magnetic intermediate states appear in the classical phase diagram, which means that (i) the non-collinear spin configurations are suppressed by the interactions in equation (1.1), and the appearance of intermediate states represents a pure quantum effect.

In conclusion, compared to some other widely discussed competing spin interactions, the isotropic three-spin exchange reveals some unique properties - such as the promotion of dimerization and linear spin configurations - which opens up an intriguing direction for future theoretical and experimental studies in the field of quantum magnetism. On the theoretical side, studies of exotic spin phases in generic 1D and higher-dimensional spin models with extra 3SE couplings constitute an interesting and promising perspective. On the experimental side, it seems that cold atoms in optical lattices, as well as the large group of alternating-spin systems could present promising routes to construct different Heisenberg spin systems with extra competing $3 \mathrm{SE}$ interactions.

\section{Acknowledgements}

I am grateful to Natalia Chepiga for the kind permission to present her phase diagrams, and to Jürgen Schnack for collaboration in the field. This research was supported by the National Science Foundation of Bulgaria (Grant KП-06-X38/6/05.12.2019).

\section{References}

1. Lacroix C., Mendels P., Mila F. (Eds.), Introduction to Frustrated Magnetism: Materials, Experiments, Theory, Springer Series in Solid-State Sciences, Vol. 164, Springer, Berlin, Heidelberg, 2011, doi $10.1007 / 978-3-642-10589-0$.

2. Läuchli A., Schmid G., Trebst S., Phys. Rev. B, 2006, 74, 144426, doi 10.1103/PhysRevB.74.144426

3. Harada K., Kawashima N., Phys. Rev. B, 2002, 65, 052403, doi:10.1103/PhysRevB.65.052403.

4. Tóth T.A., Läuchli A.M., Mila F., Penc K., Phys. Rev. B, 2012, 85, 140403(R), doi 10.1103/PhysRevB.85.140403.

5. Momoi T., Sindzingre P., Shannon N., Phys. Rev. Lett., 2006, 97, 257204, doi $10.1103 /$ PhysRevLett.97.257204

6. Smerald A., Shannon N., Phys. Rev. B, 2013, 88, 184430, doi $10.1103 /$ PhysRevB.88.184430. 
7. Bastardis R., Guihéry N., de Graaf C., Phys. Rev. B, 2007, 76, 132412, doi 10.1103/PhysRevB.76.132412.

8. Falk U., Furrer A., Güdel H.U., Kjems J.K., Phys. Rev. Lett., 1986, 56, 1956-1959, doi $10.1103 /$ PhysRevLett.56.1956

9. Falk U., Furrer A., Kjems J.K., Güdel H.U., Phys. Rev. Lett., 1984, 52, 1336-1339, doi 10.1103/PhysRevLett.52.1336.

10. Iwashita, T., Uryû N., J. Phys. Soc. Jpn., 1974, 36, 48-54, doi $10.1143 / J P S J .36 .48$

11. Furrer A., Waldmann O., Rev. Mod. Phys., 2013, 85, 367-420, doi $10.1103 /$ RevModPhys.85.367.

12. Pachos J.K., Plenio M.B., Phys. Rev. Lett., 2004, 93, 056402, doi 10.1103/PhysRevLett.93.056402

13. Pachos J.K., Rico E., Phys. Rev. B, 2004, 70, 053620, doi 10.1103/PhysRevA.70.053620

14. Tame M.S., Paternostro M., Kim M.S., Vedral V., Phys. Rev. B, 2006, 73, 022309, doi 10.1103/PhysRevA.73.022309

15. Büchler H.P., Micheli A., Zoller P., Nat. Phys., 2007, 3, 726-731, doi:10.1038/nphys678

16. Andrei N., Johannesson H., Phys. Lett. A, 1984, 100, 108-112, doi 10.1016/0375-9601(84)90675-3

17. de Vega H.J., Woynarovich F., J. Phys. A: Math. Gen., 1992, 25, 4499-4516, doi $10.1088 / 0305-4470 / 25 / 17 / 012$

18. Aladim S.R., Martins M.J., J. Phys. A: Math. Gen., 1993, 26, L529-L534, doi: 10.1088/0305-4470/26/12/009.

19. de Vega H.J., Mezincescu L., Nepomechie R.I., Phys. Rev. B, 1994, 49, 13223-13226, doi $10.1103 /$ physrevb.49.13223.

20. Bytsko A., Doikou A., J. Phys. A: Math. Gen., 2004, 37, 4465-4492, doi $10.1088 / 0305-4470 / 37 / 16 / 001$

21. Ribeiro J.A.P., Klümper A., Nucl. Phys. B, 2008, 801, 247-267, doi 10.1016/j.nuclphysb.2008.02.012.

22. Suzuki M., Prog. Theor. Phys., 1971, 46, 1337-1359, doi:10.1143/PTP.46.1337

23. Gottlieb D., Rössler J., Phys. Rev. B, 1999, 60, 9232-9235, doi:10.1103/PhysRevB.60.9232.

24. Titvinidze I., Japaridze G.I., Eur. Phys. J. B, 2003, 32, 383-393, doi $10.1140 /$ epjb/e2003-00113-8.

25. Lou P., Wu W.C., Chang M.C., Phys. Rev. B, 2004, 70, 064405, doi 10.1103/PhysRevB.70.064405

26. Zvyagin A.A., Phys. Rev. B, 2005, 72, 064419, doi 10.1103/PhysRevB.72.064419.

27. Krokhmalskii T., Derzhko O., Stolze J., Verkholyak T., Phys. Rev. B, 2008, 77, 174404, doi 10.1103/PhysRevB.77.174404.

28. Derzhko V., Derzhko O., Richter J., Phys. Rev. B, 2011, 83, 174428, doi 10.1103/PhysRevB.83.174428

29. Topilko M., Krokhmalskii T., Derzhko O., Ohanyan V., Eur. Phys. J. B, 2012, 85, 278 , doi $10.1140 /$ epjb/e2012-30359-8

30. Menchyshyn O., Ohanyan V., Verkholyak T., Krokhmalskii T., Derzhko O., Phys. Rev. B, 2015, 92, 184427, doi: 10.1103/PhysRevB.92.184427.

31. Michaud F., Vernay F., Manmana S.A., Mila F., Phys. Rev. Lett., 2012, 108, 127202, doi $10.1103 /$ PhysRevLett.108.127202

32. Michaud F., Manmana S.R., Mila F., Phys. Rev. B, 2013, 87, 140404(R), doi 10.1103/PhysRevB.87.140404.

33. Wang Z.Y., Furuya S.C., Nakamura M., Komakura R., Phys. Rev. B, 2013, 88, 224419, doi $10.1103 /$ PhysRevB.88.224419

34. Chepiga N., Affleck I., Mila F., Phys. Rev. B, 2016, 93, 241108(R), doi 10.1103/PhysRevB.93.241108

35. Chepiga N., Affleck I., Mila F., Phys. Rev. B, 2016, 94, 205112(R), doi 10.1103/PhysRevB.94.205112

36. Chepiga N., Mila F., Phys. Rev. B, 2019, 100, 104426, doi 10.1103/PhysRevB.100.10442

37. Chepiga N., Affleck I., Mila F., Phys. Rev. B, 2020, 101, 174407, doi 10.1103/PhysRevB.101.174407

38. Ivanov N.B., Ummethum J., Schnack J., Eur. Phys. J. B, 2014, 87, 226, doi:10.1140/epjb/e2014-50423-7

39. Ivanov N.B., Schnack J., J. Phys.: Conf. Ser., 2014, 558, 012015, doi 10.1088/1742-6596/1186/1/102014.

40. Ivanov N.B., Petrova S.I., Schnack J., Eur. Phys. J. B, 2016, 89, 121, doi $10.1140 /$ epjb/e2016-70057-y

41. Ivanov N.B., Schnack J., J. Phys.: Conf. Ser., 2019, 1186, 012014, doi:10.1088/1742-6596/558/1/012015.

42. Kolezhuk A.K., Schollwöck U., Phys. Rev. Lett., 1996, 77, 5142-5145, doi 10.1103/PhysRevLett.77.5142

43. Affleck I., Haldane F.D.M., Phys. Rev. B, 1987, 36, 5291-5300, doi 10.1103/PhysRevB.36.5291.

44. Landee Ch.P., Turnbull M.M., Eur. J. Inorg. Chem., 2013, 2013, 2266-2285, doi 10.1002/ejic.201300133

45. Ivanov N.B., Richter J., Phys. Rev. B, 2004, 69, 214420, doi 10.1103/PhysRevB.69.214420

46. Lieb E.H., Mattis D.C., J. Math. Phys., 1962, 3, 749-751, doi:10.1063/1.1724276

47. Oshikawa M., Yamanaka M., Affleck I., Phys. Rev. Lett., 1997, 78, 1984-1987, doi $10.1103 /$ PhysRevLett.78.1984

48. Shimokawa T., Nakano H., J. Korean Phys. Soc., 2013, 63, 591-595, doi $10.3938 /$ kkps.63.591

49. Furuya Sh.C., Giamarchi Th., Phys. Rev. B, 2014, 89, 205131, doi 10.1103/PhysRevB.89.205131

50. Anderson P.W., Phys. Rev. B, 1952, 86, 694-701, doi 10.1103/PhysRev.86.694

51. Schnack J., Luban M., Phys. Rev. B, 2000, 63, 014418, doi $10.1103 /$ PhysRevB.63.014418 


\section{Гайзенберґові спінові ланцюжки з додатковими ізотропними триспіновими обмінними взаємодіями}

\section{Н.Б. Іванов}

Інститут фізики твердого тіла, Болгарська академія наук, Царіградско шосе 72, Софія 1784, Болгарія

$J_{1}-J_{3}$ Гайзенберґові спінові моделі з взаємодією найближчих сусідів $\left(J_{1}\right)$ та додатковою ізотропною триспіновою взаємодією $\left(J_{3}\right)$ залишаються менш дослідженими, хоча такі типи конкуруючих обмінних членів можуть природно виникати з різних причин, включаючи розвинення сильного зв'язку мультиорбітальної моделі Габарда. Нижче ми представляємо короткий огляд нещодавніх досліджень у цій області з наголосом на характеристики розмаїття квантових фаз, які підтримуються декількома типовими одновимірними $J_{1}-J_{3}$ моделями Гайзенберґа з однорідними і змішаними спінами. Оскільки додатні $\left(J_{3}>0\right)$ триспінові взаємодії ведуть до формування локальних квантових димерів, $J_{1}-J_{3}$ спінові моделі зазвичай зазнають певної спонтанної димеризації при збільшенні $J_{3}$. Справді, виявляється, що встановлені димерні фази у спін- $S J_{1}-J_{3}$ Гайзенберґових ланцюжках $\left(S>\frac{1}{2}\right)$ служать повними аналогіями відомої щілинної димерної фази Маджумдара-ґоша у спін- $\frac{1}{2}$ Гайзенберґовому ланцюжку з взаємодією між наступними після найближчих сусідами. Такі ж димеризації виявлено у $J_{1}-J_{3}$ ланцюжках зі змішаними спінами- $(S, \sigma)$ за умови, що спін комірки $S+\sigma=$ integer, в той час як для пів-цілого спіна формування локальних димерів веде до безщілинних станів спінової рідини. $J_{1}-J_{3}$ ланцюжки зі змішаними спінами також передбачають деякі типові приклади спінових моделей, які реалізують так звані магнітні фази Ліба-Маттіса.

Ключові слова: спінові ланцюжки, квантові спінові фази, триспінові взаємодії 